\title{
Experimental Study on Flow Field Characteristics of a Free Circular Swirling Jet
}

\author{
Shams Sourav and Md. Abu Taher Ali
}

\begin{abstract}
Flow field characteristics of a free circular swirling jet are investigated in the present study considering three different swirl numbers $0.38,0.56$ and 0.80 . The experiment is carried out in an air jet facility for Reynolds numbers 16300,34400 and 49200 . Pressure probe method is used for 3-hole wedge shaped yaw meter to measure and calculate the data. Mean flow velocity along with centerline mean velocity decay are analyzed where swirl changes the instinctive properties of circular jet and demolishes the potential core. Swirl motion creates negative flow field which changes the static pressure and kinetic energy distribution enormously. As a result, flow diverges earlier, and surrounding air enters into the flow field more rapidly which enhances the mixing performance of the jet.
\end{abstract}

Keywords - Kinetic Energy, Mean Velocity, Static Pressure, Swirling Jet, Turbulent Flow.

\section{INTRODUCTION}

Enhancing the mixing performance of a jet flow is one of the most engaging research affairs due to a wide range of industrial applications involving gas turbine combustion, cyclone separator, chemical reactor, burner, cooling and so on. Introducing swirl in the jet is not speculating anymore rather analyzing its turbulent characteristics for improved mixing and flame stabilization becomes captivating for the researchers for its intricate and unpredictable flow structure. Swirl exhibits greater mixing efficiency over a laminar flow.

From last few decades, many investigations are being conducted both numerically and experimentally to develop the swirl flow structure and characteristics. For instances, Shiri and George [1] experimentally investigated incompressible swirl jets for swirl number 0.15 and 0.25 up to jet exit location 50D by using Laser Doppler Anemometry (LDA). They noticed that velocity, turbulent intensity, centerline decay and growth rates are identical to nonswirling jets for swirl number 0.15 . But velocity and turbulent intensity collapse very early for swirl number 0.25 . Dynamic Mode Decomposition of a swirl jet for high Re and swirl number was analyzed by Lambardi et al. [2] using PIV method where they observed that precessing vortex core is present and dynamically stable in terms of frequency, growth rate and spatial patterns. Free and impinging swirling jets were studied by Aleekseenko et al. [3] for a fixed $\mathrm{Re}=8900$ and varying swirl number 0 to 1 . They used stereo PIV method and found that, impinging plate plays a great role for characterizing flow field where impact is greatest for swirl

Submitted on December 21, 2021

Published on January 14, 2022.

Shams Sourav, Department of Aeronautical Engineering, Military Institute of Science and Technology (MIST), Bangladesh.

(e-mail: sourav.shams@gmail.com) number 0.41 . They also noticed that large recirculation region is not present in case of free jet and turbulent kinetic energy is higher for impinging jet. Recalcati et al. [4] used PIV method to measure entrainment rate in the near field for swirl number ranging from 0 to 1.5 and Re 24400 to 30700 . They concluded that rate of entrainment increases non-linearly in the downstream direction and mixing is enhanced with the increase of swirl number. Vortex breakdown in the flow structure was studied by Vanierschot [5] for annular swirling jets at transition region for $\mathrm{Re}=460$ and swirl number 0.55 . Time resolved stereoscopic PIV with combined proper orthogonal decomposition (POD) and spectral proper orthogonal decomposition (SPOD) was used to analyze the flow structures. He found a vortex breakdown bubble along with precessing vortex core (PVC) in the inner shear layer where mixing is larger compared to outer shear layer. A PVC is also formed in outer shear layer, and it revolves in opposite direction to the swirl flow. Hossain et al. [6] investigated the flow characteristics of swirling confined jet for varying swirl numbers with a fixed $\mathrm{Re}=53000$. They observed that mixing phenomenon increases with the increase of swirl number, but swirl jet loses its characteristics inside the confined area and flow becomes more organized after coming out of confined region. Mudkavi [7] numerically analyzed the phenomenon of vortex breakdown and found that it is highly unstable and very unpredictable. Moreover, it is independent of Re beyond $\operatorname{Re}=100$ but depends on Rossby number $\left(R_{o}=u^{*} / r^{*} \Omega^{*}\right)$ defined by Spall et al. [8]. Shear layer stability of swirling jet was studied by Martin and Meiburg [9] using potential theory where they derived the expressions for growth rate and propagation velocity of both axisymmetric and helical waves. They noticed that centrifugally stable flow becomes unstable by sufficiently small Kelvin- Helmholtz waves. This instability dominates for long axisymmetric waves and short waves converge to a plane Kelvin-Helmholtz instability which creates azimuthal vortices. Cozzi et al. [10] investigated the effects of swirl intensity on isothermal free swirling jet by stereoscopic PIV method for swirl number 0.4 , $0.76,1,1.26$ and 1.78 at a constant $\operatorname{Re}=21800$. They found the presence of central recirculation zone (CRZ) for high swirl numbers which enhances the mixing of air/fuel for combustion and produces a low velocity region for flame stabilization. They also observed that spread rate increases and vortex bubble rises axially with the swirl number. Abry et al. [11], Nayeem et al. [12], Escudier et al. [13] and other

Md. Abu Taher Ali, Department of Aeronautical Engineering, Military Institute of Science and Technology (MIST), Bangladesh. (e-mail: matali@ae.mist.ac.bd). 
researchers studied swirl jet both numerically and experimentally.

However, very few researchers experimentally focused on swirl effects on jet flow with varying Re. This experiments attributes to the impact of swirl on turbulent characteristics of jet which is anticipated to improve the research activities on present field.

\section{EXPERIMENTAL DETAILS}

The experiment was carried out in a circular air jet flow facility shown in Fig. 1, which has an inlet diameter of 300 $\mathrm{mm}$ with an axial fan unit (consists of two axial fan), settling chamber, reduced, flow straightener, flow stabilizer, vortex breaker, reducer and finally a discharge pipe nozzle of exit diameter $\mathrm{D}=20 \mathrm{~mm}$ which includes a swirl generator.

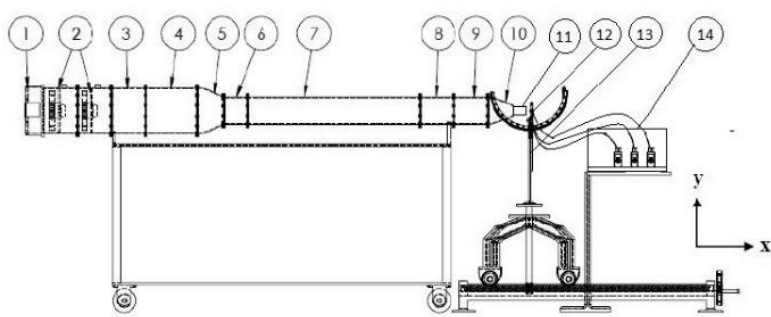

Fig. 1. Air jet nozzle setup, (1) Flow Controller, (2) Fan Unit, (3) Guide Vane, (4) Settling Chamber, (5) Reducer, (6) Flow Straightener,

(7) Flow Stabilizer Pipe, (8) Vortex Breaker, (9) Discharge Pipe, (10) Reducer, (11) Discharge Nozzle, 12) Yaw meter,

13) Traversing Mechanism, 14) Pressure Transducer.

To produce swirl flow of three different swirl numbers (S) of $0.38,0.56$ and 0.80, a spiral guide vane shown in Fig. 2 is used having three different swirl angles $(\varphi)$ of 30, 40 and 50 degrees respectively. Following relationship is established by Gupta et al. [14] to determine swirl number for a hubless swirler.

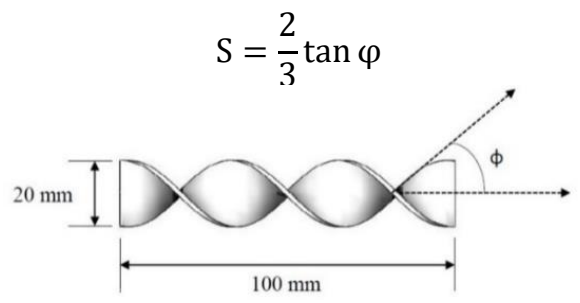

Fig. 2. Swirl Generator.

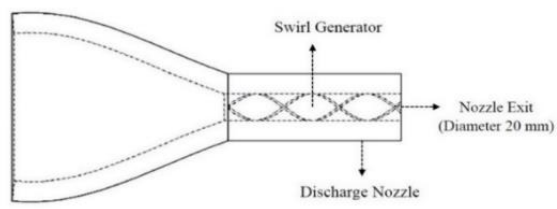

(a) $2 \mathrm{D}$ view (b) Isometric view

Fig. 3. Geometry of swirl jet nozzle (a) 2D view and (b) Isometric View.

The geometry of the swirling pipe jet nozzles is shown in Fig. 3. The pipe jet nozzle has the capability of generating maximum flow velocity of $36 \mathrm{~m} / \mathrm{s}$ at the nozzle exit without spiral guide vane installed. A flow controller is used at the inlet of the nozzle to regulate the flow. The experiment is performed at three different Reynolds numbers $(\mathrm{Re}=16300$, 34400 and 49200). To measure the flow velocity and direction, pressure probe method [15] was used. A calibrated three-hole wedge shaped yaw meter and three dual input differential pressure transducers of range $\pm 13.79 \mathrm{kPa}$ with 0.01 resolution and $\pm 0.3 \%$ accuracy was utilized for data extraction. A three dimensional traversing mechanism was used to mount and move the probe at different longitudinal $(\mathrm{x})$, transverse $(\mathrm{y})$ and lateral $(\mathrm{z})$ directions. Co-ordinate system of the nozzle is shown in Fig. 4. Non-dimensional data are used for plotting only in the positive directions of all three axis due to symmetrical properties of twin jet. Exit mean velocity $\left(\mathrm{U}_{\mathrm{co}}\right)$, dynamic pressure $\left(\mathrm{P}_{\mathrm{d}}\right)$ and kinetic energy at jet exit $\left(\mathrm{K}_{\mathrm{o}}\right)$ are used to non-dimensionalize stream wise mean velocity $\left(\mathrm{U}_{\mathrm{x}}\right)$, static pressure $\left(\mathrm{P}_{\mathrm{s}}\right)$ and mean kinetic energy $(\mathrm{K})$ respectively. Ambient temperature and pressure were constantly observed and recorded during the experiment.

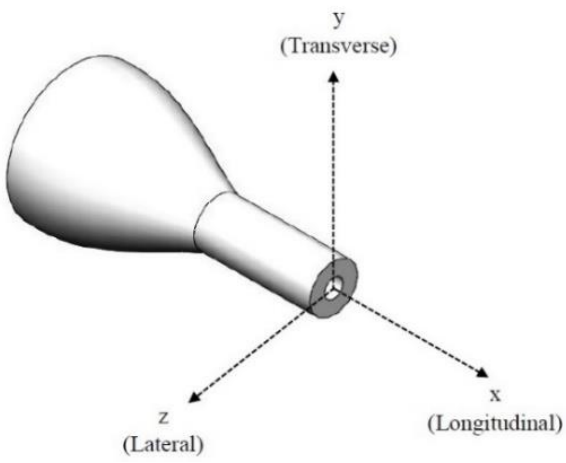

Fig. 4. Co-ordinate system of the nozzle.

\section{RESUlts AND DisCUSSION}

\section{A. Centerline Mean Velocity Decay}

Fig. 5 and Fig. 6 show the centerline velocity decay at different $\mathrm{Re}$ and swirl number respectively. Along the centerline, velocity maxima are obtained for lower swirl number and as it increases, centerline mean velocity tends to be more negative at the near field of jet exit. As Re increases, maximum velocity in the centerline also increases and turns towards upstream direction.

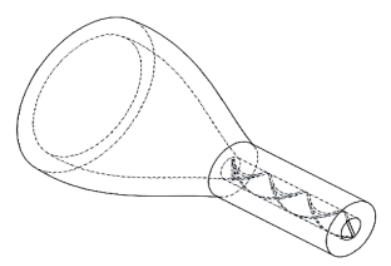




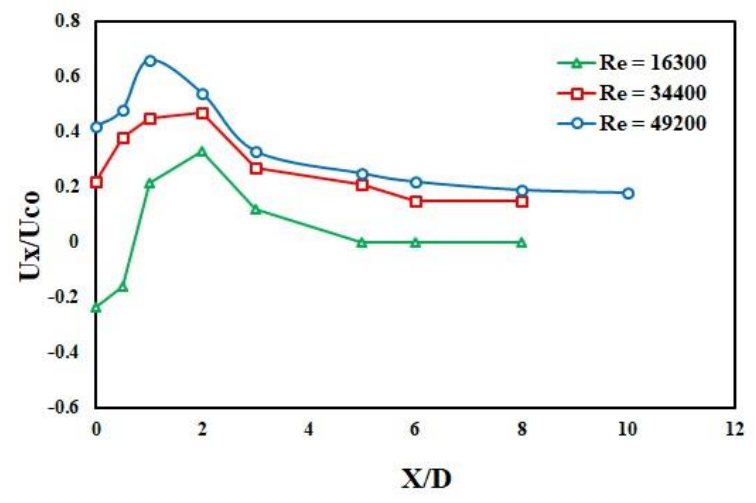

(a) Swirl number, $\mathrm{S}=0.38$

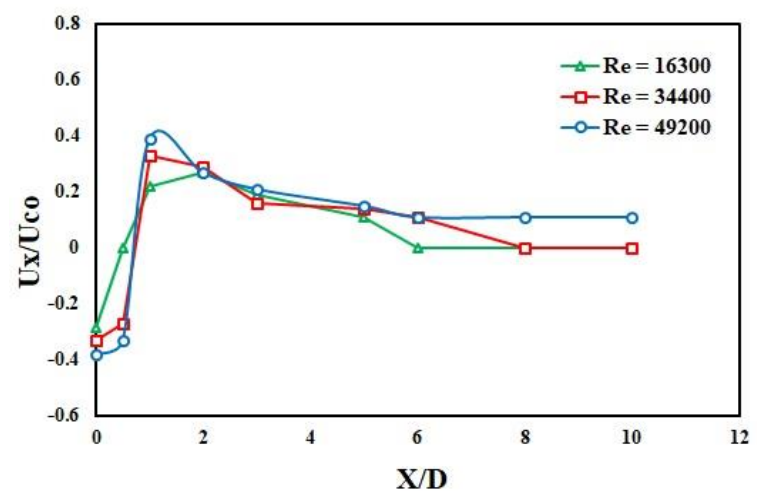

(b) Swirl number, $\mathrm{S}=0.56$

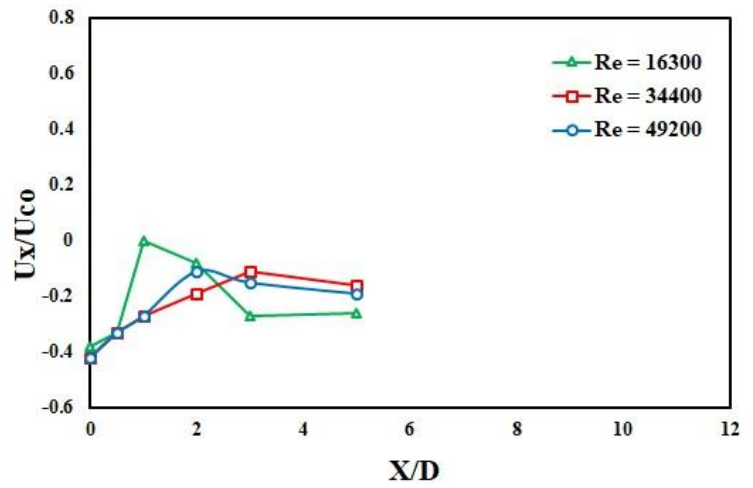

(c) Swirl number, $\mathrm{S}=0.80$

Fig. 5. Centerline mean velocity decay for different Re at (a) $S=0.38$, (b) $\mathrm{S}=0.56$ and (c) $\mathrm{S}=0.80$.

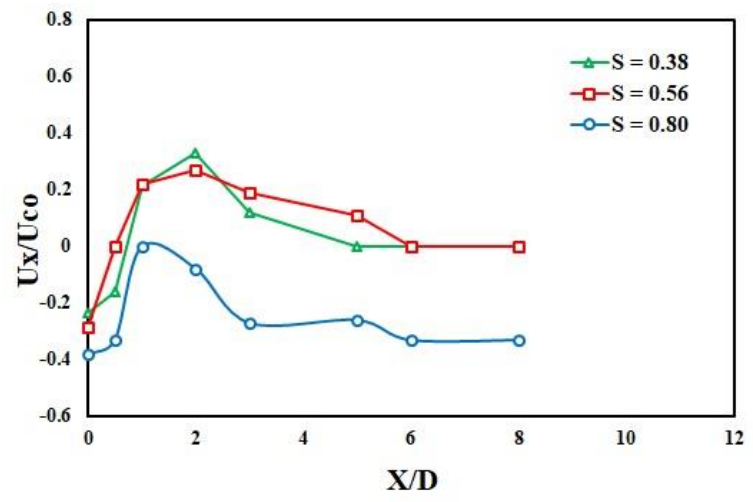

(a) $\operatorname{Re}=16300$

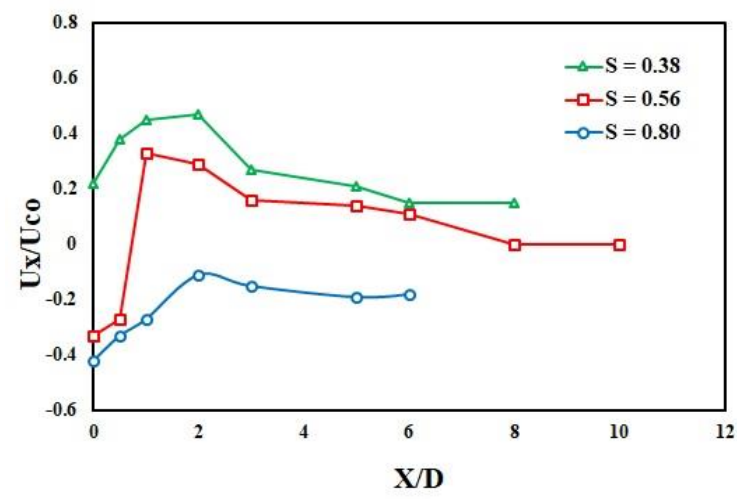

(b) $\mathrm{Re}=34400$

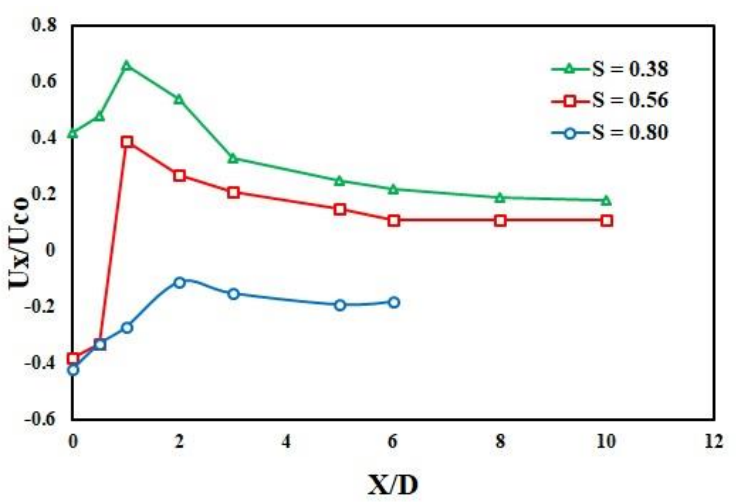

(c) $\operatorname{Re}=49200$

Fig. 6. Centerline mean velocity decay for different swirl numbers at (a) $\operatorname{Re}=16300$, (b) $\operatorname{Re}=34400$ and (c) $\operatorname{Re}=49200$

\section{B. Mean Velocity Profiles}

Fig. 7, Fig. 8, and Fig. 9 represent the mean velocity profiles of swirl jets for three different swirl numbers $(0.38$, 0.56 and 0.80$)$ at three varying Reynolds numbers $(\operatorname{Re}=$ 16300,34400 and 49200) respectively. It is observed that maximum mean velocity moves from jet centerline towards the jet periphery which is also previously found by Nayeem et al. [12]. Velocity maxima increases in the flow field with Re for all three swirl numbers. Recirculation region is also evident in the flow field and with the rise of swirl number, flow tends to show more negative velocities for all $\mathrm{Re}$ considered. Strong velocity fluctuation is noticed from X/D = 1 and onwards for swirl numbers 0.38 and 0.56 . Whereas, for swirl number 0.80 , this velocity fluctuation tends to reduce. Flow becomes self-similar in the far field and as swirl number increases, self-similar region shifts towards the upstream direction. This may be due to increased entrainment rate of surrounding fluids and centrifugal flow produced by the swirl generator at the nozzle exit. 


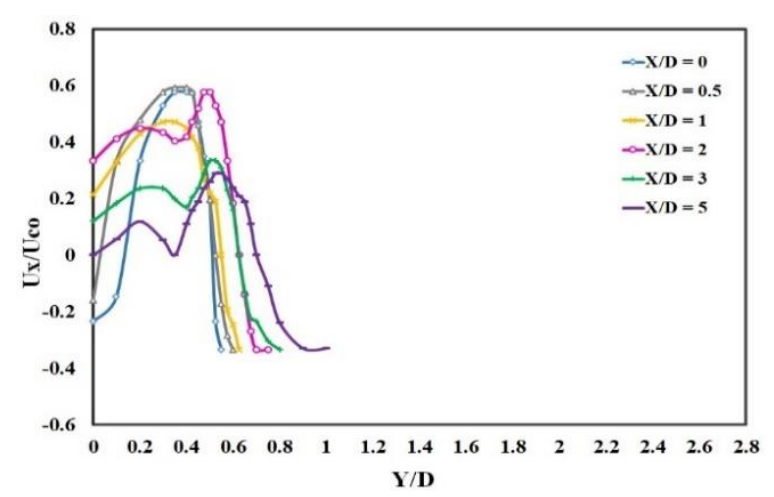

(a) $\operatorname{Re}=16300$

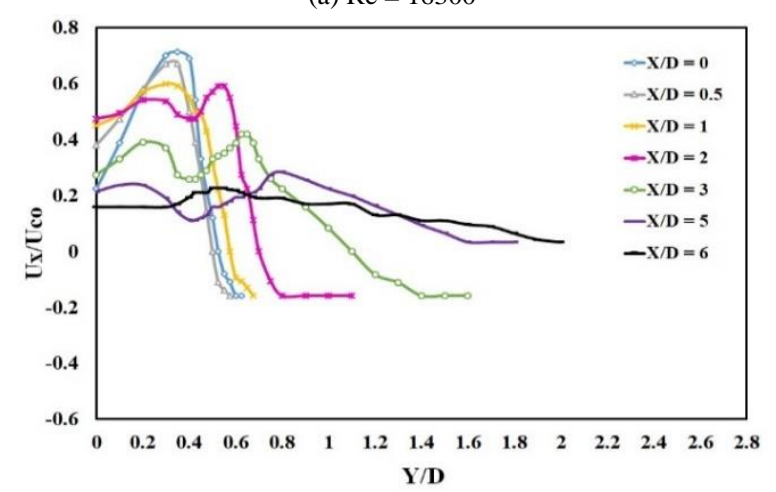

(b) $\operatorname{Re}=34400$

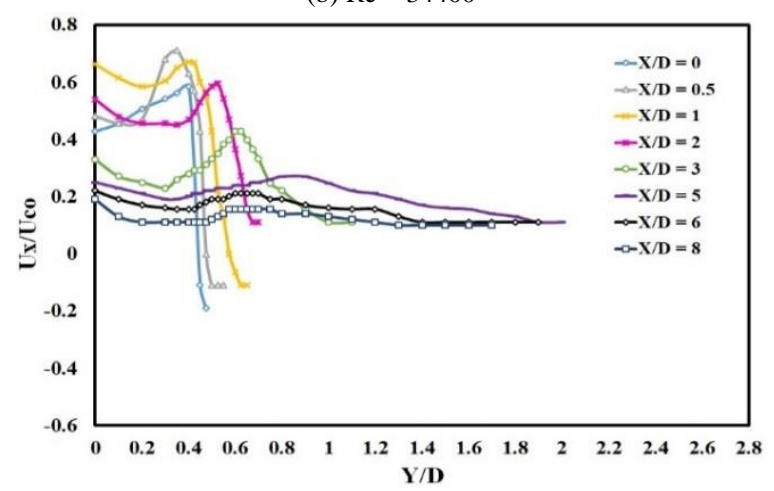

(c) $\operatorname{Re}=49200$

Fig. 7. Mean velocity profile for swirl number, $S=0.38$ at (a) $R e=16300$, (b) $\operatorname{Re}=34400$ and (c) $\operatorname{Re}=49200$.

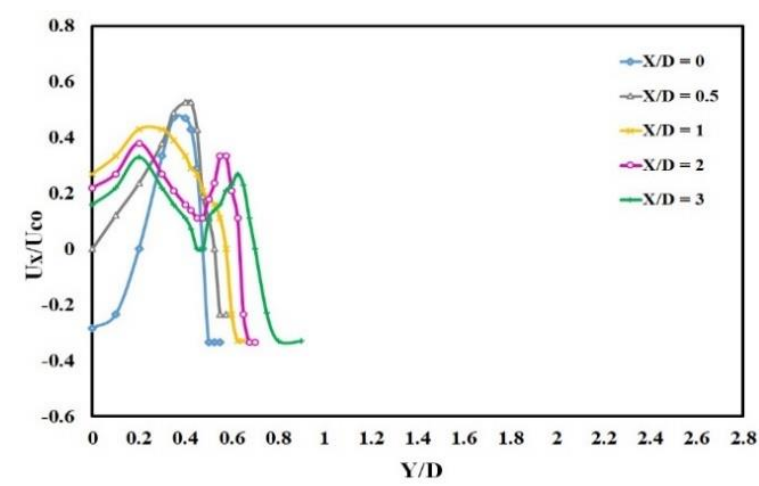

(a) $\operatorname{Re}=16300$

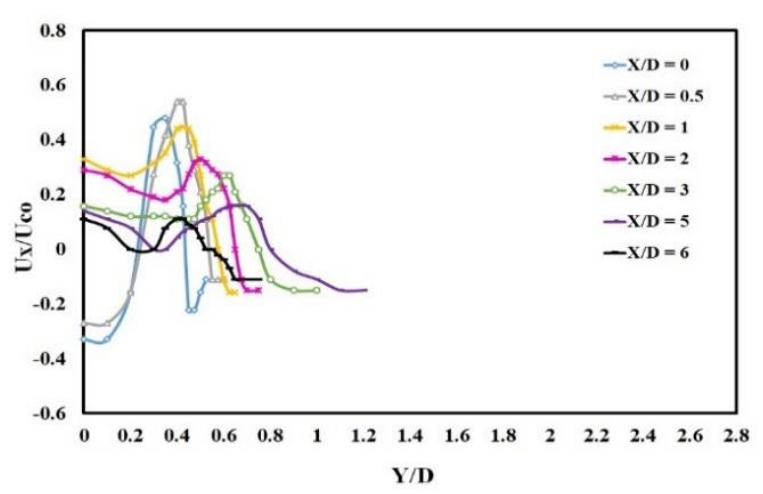

(b) $\operatorname{Re}=34400$

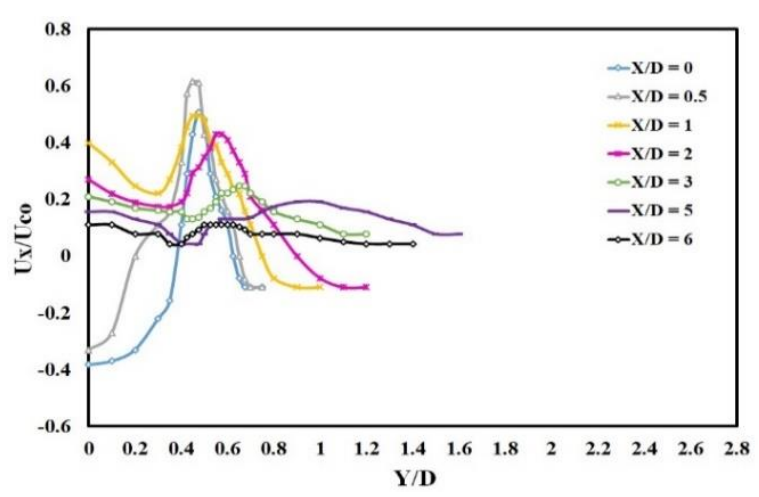

(c) $\operatorname{Re}=49200$

Fig. 8. Mean velocity profiles for swirl number, $S=0.56$ at (a) $R e=16300$, (b) $\operatorname{Re}=34400$ and (c) $\operatorname{Re}=49200$.

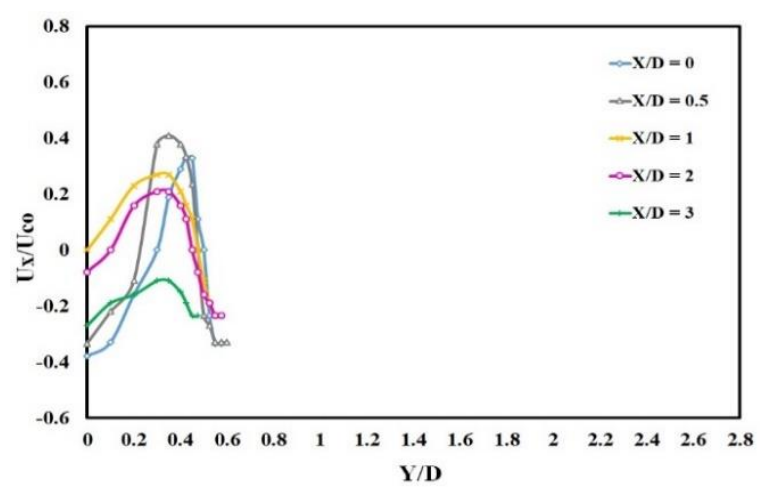

(a) $\operatorname{Re}=16300$

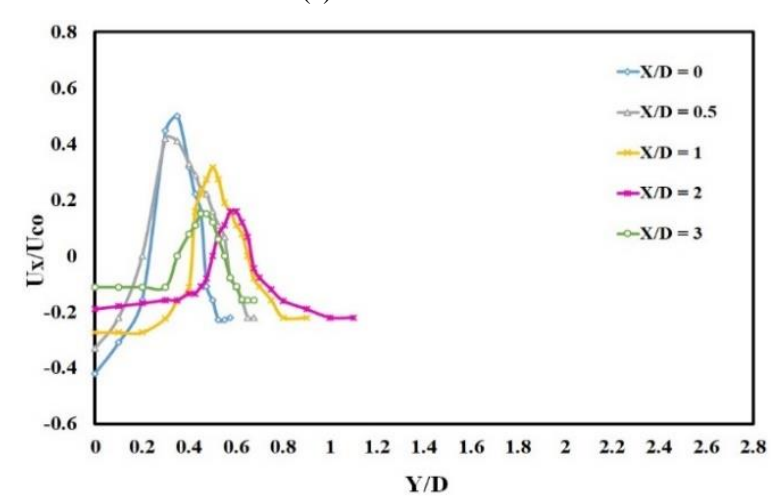

(b) $\operatorname{Re}=34400$ 


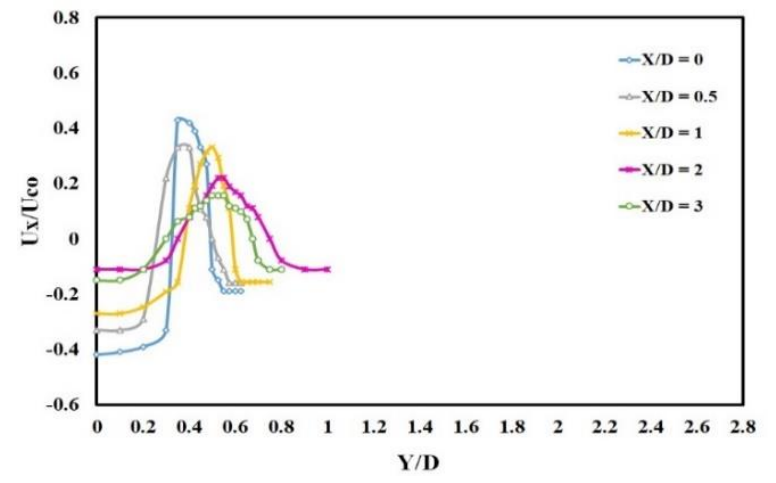

(c) $\operatorname{Re}=49200$

Fig. 9. Mean velocity profiles for swirl number, $S=0.80$ at (a) $\operatorname{Re}=16300$, (b) $\operatorname{Re}=34400$ and (c) $\operatorname{Re}=49200$.

\section{Static Pressure Distribution}

Static pressure distributions along the centerline are shown in Fig. 10 and Fig. 11 for three different swirl numbers and Re respectively. With the increase $\mathrm{Re}$, static pressure also increases. Rapid progression of static pressure is observed in the near field for higher swirl number. Negative pressure is found in this region and this negative pressure grows with upturn of swirl number. In the near field after $\mathrm{X} / \mathrm{D}=1$, static pressure becomes self-similar, and flow tends to show asymptotic properties in the far field for all three Re and swirl numbers.

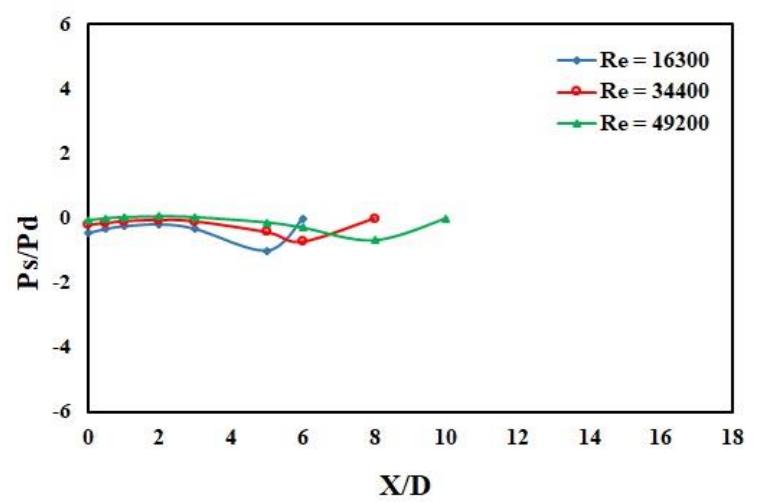

(a) Swirl number, $\mathrm{S}=0.38$

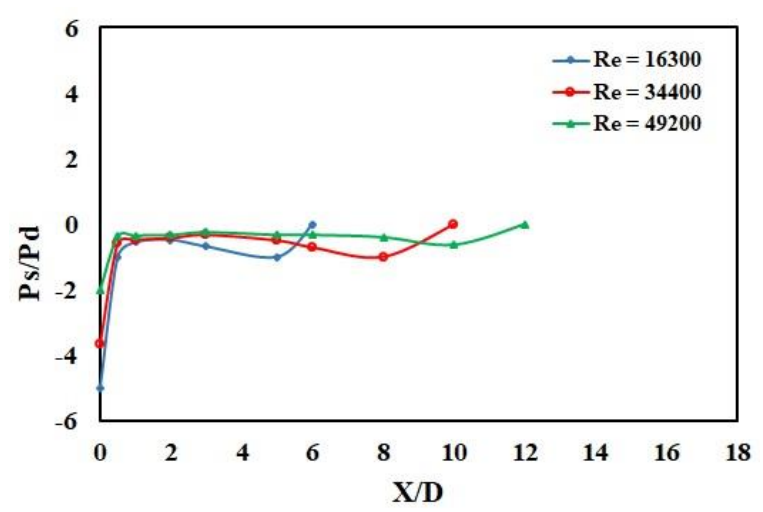

(b) Swirl number, $\mathrm{S}=0.56$

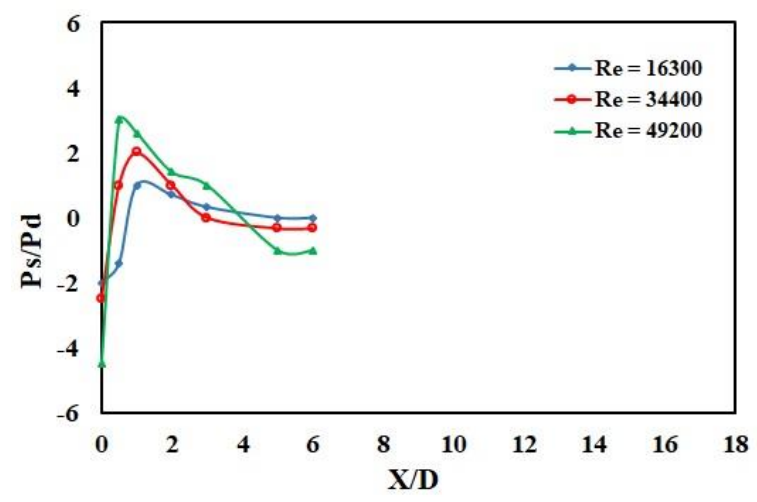

(c) Swirl number, $\mathrm{S}=0.80$

Fig. 10. Static pressure distribution along the centerline at (a) $\mathrm{S}=0.38$, (b) $\mathrm{S}=0.56$ and (c) $\mathrm{S}=0.80$.

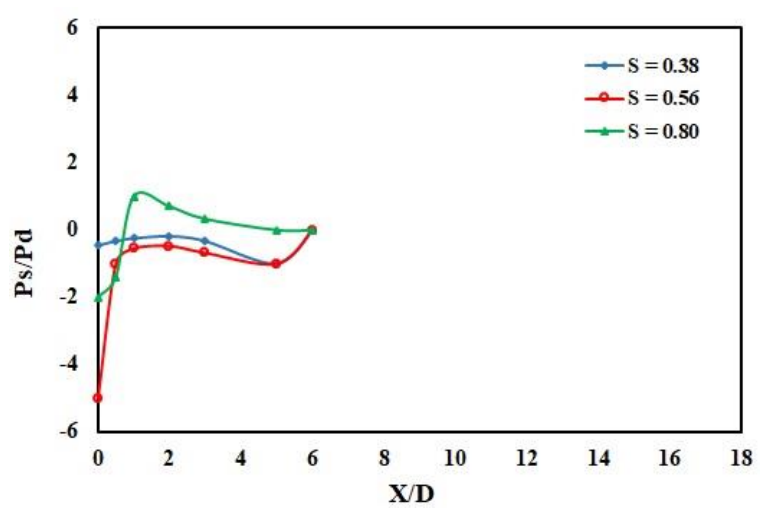

(a) $\operatorname{Re}=16300$

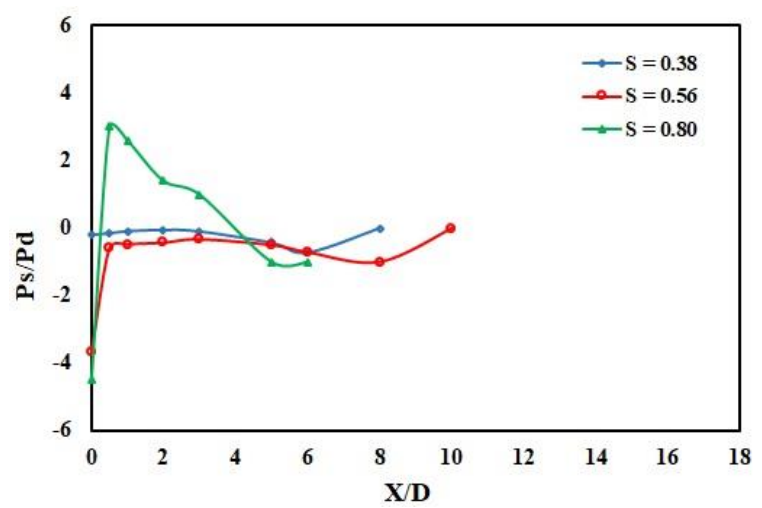

(b) $\operatorname{Re}=34400$

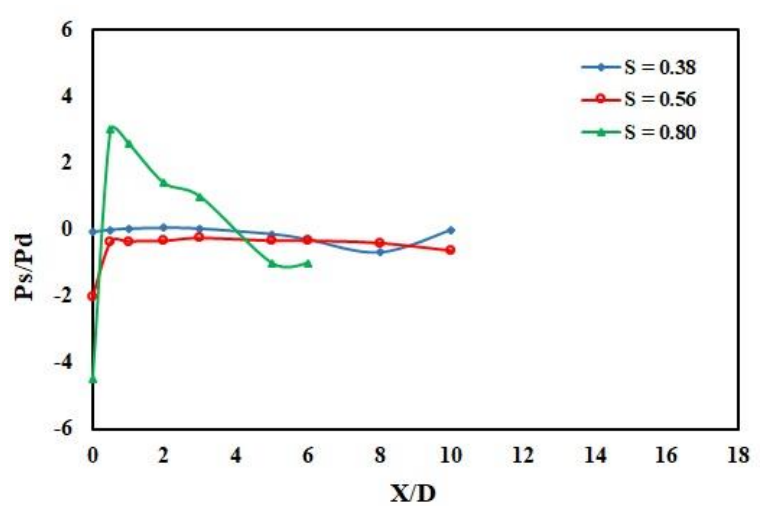

(c) $\mathrm{Re}=49200$

Fig. 11. Static pressure distribution along the centerline at (a) $\operatorname{Re}=16300$, (b) $\operatorname{Re}=34400$ and (c) $\operatorname{Re}=49200$. 


\section{Mean Kinetic Energy Distribution}

Fig.12 and Fig.13 illustrate the mean kinetic energy distribution along the centerline. Results show that kinetic energy increases with Re. For low swirl number (0.38), kinetic energy is higher in the near field up to $\mathrm{X} / \mathrm{D}=2$ (approx.) for all Re. With the increase of swirl number, kinetic energy starts to dissipate early in the downstream direction. This early attenuation of energy happens due to improved mixing of surrounding fluids for high swirl number. Although there is an insignificant variation in the rate of energy dissipation with varying Re.

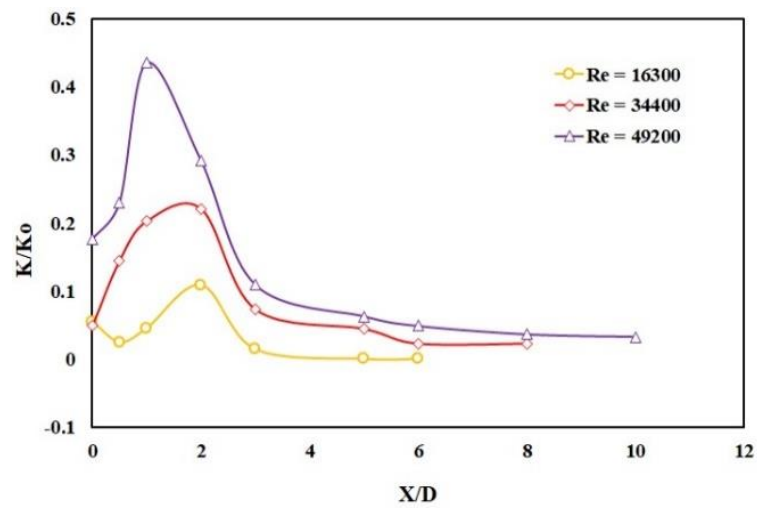

(a) Swirl number, $\mathrm{S}=0.38$

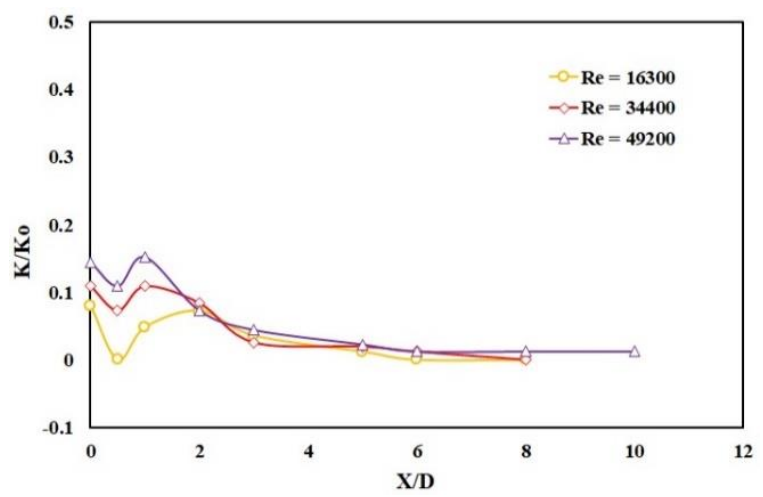

(b) Swirl number, $\mathrm{S}=0.56$

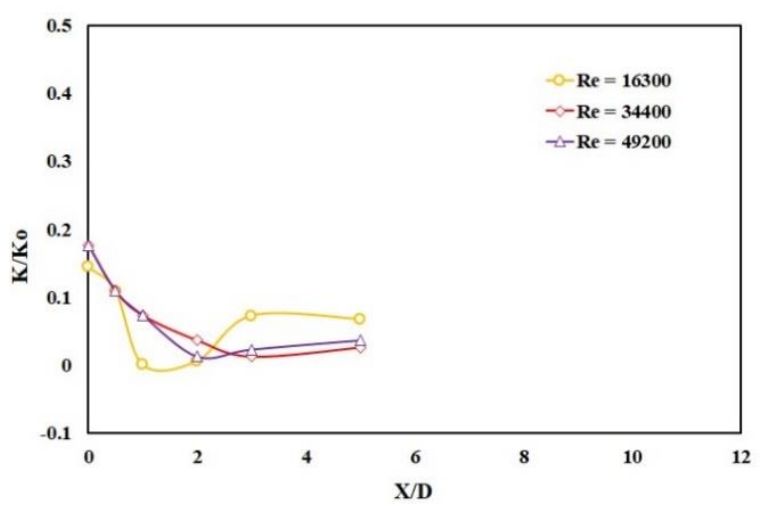

(c) Swirl number, $\mathrm{S}=0.80$

Fig. 12. Kinetic energy distribution along the centerline at (a) $\mathrm{S}=0.38$, (b) $\mathrm{S}=0.56$ and (c) $\mathrm{S}=0.80$.

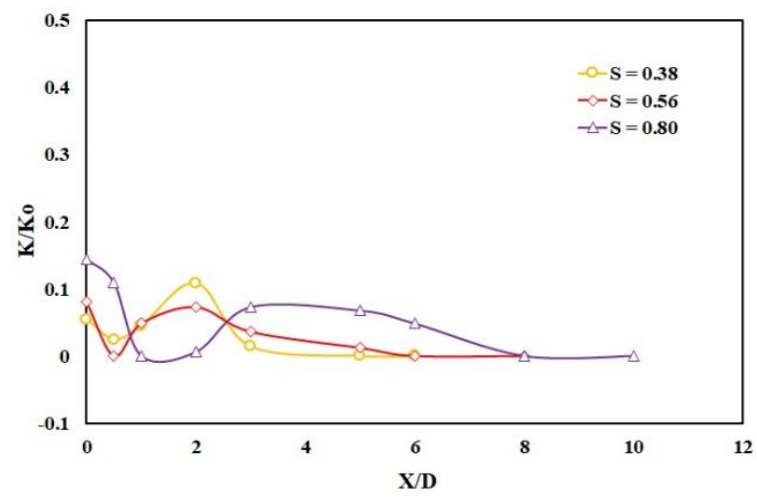

(a) $\mathrm{Re}=16300$

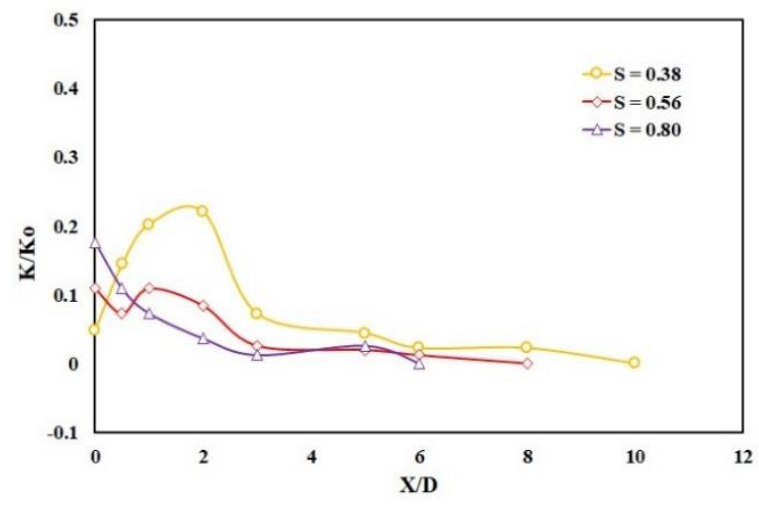

(b) $\operatorname{Re}=34400$

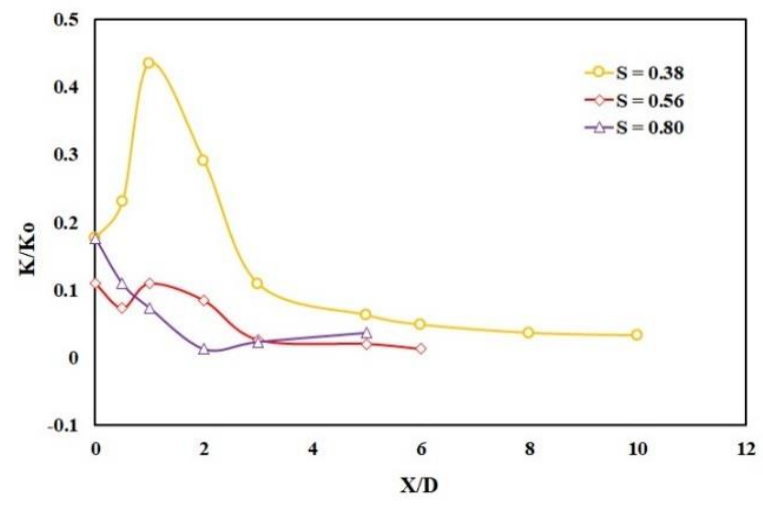

(c) $\operatorname{Re}=49200$

Fig. 13. Kinetic energy distribution along the centerline at (a) $\operatorname{Re}=16300$, (b) $\operatorname{Re}=34400$ and (c) $\operatorname{Re}=49200$.

\section{CONCLUSION}

Flow fields of a circular swirling jet are studied in this experiment at different Reynolds numbers and swirl numbers. A significant impact is noticed for characterizing the circular jet flow field. Velocity maxima shifts from the jet centerline and recirculation region is evident in the near field where negative velocity exists and this negative velocity grows with the increase of swirl number. Intense mean velocity variation is found in the near field (X/D = 1 and onwards approx.) for swirl number up to 0.56 but this fluctuation leans to decrease in swirl number 0.80 . Velocity decay in the centerline is highly unstable and unpredictable. Higher velocity is found in the centerline for lower swirl number and maximum centerline velocity shifts towards the upstream direction in the near field. Static pressure distribution is also studied 
where rapid increase of static pressure is observed in the near field for higher swirl number. After $\mathrm{X} / \mathrm{D}=1$, static pressure becomes self-similar and exhibits asymptotic property for all swirl number and Re. Mean kinetic energy is found to be higher in the near field region up to $\mathrm{X} / \mathrm{D}=2$ (approx.) which is independent of Re. Energy dissipation starts early when swirl number increases which occurs due to improved entrainment rate and mixing of surrounding fluids.

\section{ACKNOWLEDGMENT}

Authors would like to express the heartiest gratitude to the previous researchers for their relentless effort to develop the whole test facility. They acknowledge the continuous supports of all the faculties of Aeronautical Engineering Department, MIST. They are also thankful to the aerodynamics and machine lab personnel for their tireless assistance.

\section{CONFLICT OF INTEREST}

Authors declare that they do not have any conflict of interest.
[13] Escudier, M. P., Nickson, A. K. and Poole, R. J. Influence of outlet geometry on strongly swirling turbulent flow through a circular tube. Physics of Fluids, 2006; 18(12):1-18.

[14] Gupta, A. K., Lilley, D. G. and Syred, N. Swirl flows. Abacus Press, Tunbridge Wells; 1984.

[15] Bryer, D. W. and Pankhrust, R. C. Pressure probe methods for determining wind speed and flow direction. National Physical Laboratory, London; 1971.

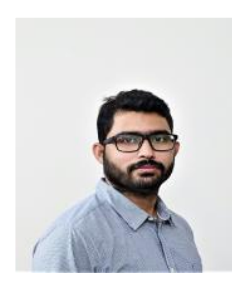

Shams Sourav is an aircraft engineer and pursuing his MSc. from the Department of Aeronautical Engineering in Military Institute of Science and Technology (MIST), Dhaka, Bangladesh. He completed his BSc in Aeronautical Engineering from MIST in 2014.

He has over 6 years of professional experience in aviation and is actively involved in research in the field of fluid mechanics and aerodynamics.

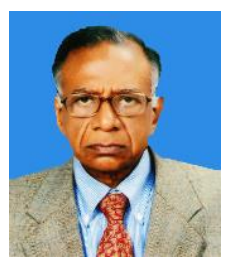

Md. Abu Taher Ali is a professor of Department of Aeronautical Engineering (AE) in Military Institute of Science and Technology (MIST), Dhaka, Bangladesh. He completed his $\mathrm{PhD}$ in Mechanical Engineering from Imperial college, University of London, England (1980). He pursued his Masters of Science in Mechanical Engineering from Bangladesh University of Engineering and Technology, Dhaka, Bangladesh (1972). He completed his Bachelor of Engineering in Mechanical Engineering with $1^{\text {st }}$ class, $4^{\text {th }}$ positionfrom East Pakistan University of Engineering and Technology, Dhaka (1966).

He has over 50 years of dynamic teaching and research experience in the corresponding department.

\section{REFERENCES}

[1] Shiri, A. F. and George, W. K. An experimental study of the far field of incompressible swirling jet. Proceedings of $36^{\text {th }}$ AIAA Fluid Dynamics Conference and Exhibit; 2006 Jun 5-8; San Francisco, California.

[2] Lombardi, S., Bizan, K., Coghe, A., Cozzi, F. and Continillo, G. DMD analysis of experimental PIV data of a swirled jet. Proceedings of $25^{\text {th }}$ International Colloquium on the Dynamics of Explosions and Reactive Systems; 2015 Aug 2-7; Leeds, UK.

[3] Alekseenko, S. V., Bilsky, A. V., Dulin, V. M. and Markovich, D. M. Experimental investigation of free and impinging swirling turbulent jets with different inflow conditions. Proceedings of $5^{\text {th }}$ International Symposium on Turbulence and Shear Flow Phenomena; 2007 Aug 27. 29; Munich, Germany.

[4] Recalcati, M., Cozzi, F. and Coghe, A. Measurement of entrainment rate in the initial region of swirling jets. Proceedings of the $X X X V$ Meeting of the Italian Section of the Combustion Institute; 2012 Oct 10-12; Milano, Italy.

[5] Varnierschot, M. On the structur of vortex breakdown in swirling jets in the transitional regime. Proceedings of the $15^{\text {th }}$ International Conference on Fluid Control, Measurements and Visualization; 2019 May 27-30; Naples, Italy.

[6] Hossain, M. M., Nayeem, M. H. K. and Ali, M. A. T. Study of the flow characteristics of swirling confined jet. International Journal of Research and Scientific Innovation, 2019; 6(12):209-212.

[7] Mudkavi, V. Y. The phenomenon of vortex breakdown. Proceedings of the Fluid Dynamics Symposium; 1993 Jul 9; Sikkim, India.

[8] Spall, R. E., Gatski, T. B. and Grosch, C. E. On a criterion for vortex breakdown. NASA CR-178232; 1987.

[9] Martin, J. E. and Meiburg, E. On the stability of the swirling jet shear layer. Physics of Fluids, 1993; 6(1):424-428.

[10] Cozzi, F. Sharma, R. Coghe, A. and Arzuffi, F. An experimental investigation on isothermal free swirling jet. Proceedings of the XXXVIII Meeting of the Italian Section of the Combustion Institute; 2015 Sept 20-23; Lecce, Italy.

[11] Abry, P., Fauve, S., Flandrin, P. and Laroche, C. Analysis of pressure fluctuations in swirling turbulent flows. Journal of Physics, 1994 4(5):725-733.

[12] Nayeem, M. H. K., Rahman, R., Nayem, N. M. and Ali, M. A. T. Experimental study of circular swirling jet. Proceedings of $8^{\text {th }}$ International Conference on Thermal Engineering; 2019 Dec 19-21; Dhaka, Bangladesh. 